Conclusions The prevalences of LETP and LCSI are high in the Americas. Public health strategies should aim to create surveillance systems of syphilis in PLWHA and assure annual screening and timely treatment.

\section{P040 IMPLEMENTING A RAPID SEXUAL HEALTH TESTING, DIAGNOSIS AND TREATMENT SERVICE: QUALITATIVE EVALUATION}

1J Horwood*, ${ }^{1} \mathrm{~A}$ Lorenc, ${ }^{1} \mathrm{E}$ Brangan, ${ }^{1} \mathrm{~J}$ Kesten, ${ }^{2} \mathrm{M}$ Croft, ${ }^{2} \mathrm{M}$ Clarke, ${ }^{1,2} \mathrm{P}$ Horner. ${ }^{1}$ University of Bristol, Bristol, UK; ${ }^{2}$ Unity Sexual Health, Bristol, UK

\subsection{6/sextrans-2021-sti.186}

Background Unity Sexual Health in Bristol UK, re-designed its service to improve access and delivery of care. This includes a Panther (Hologic Inc) system at the point of care to provide rapid STI tests, allowing Nucleic acid amplification testing results for STIs including gonorrhoea and chlamydia to be available within four hours. Previously patients waited over a week for results.

Methods A qualitative evaluation running alongside the implementation of the new service, to understand experiences, and inform its iterative development. A total of 21 members of staff and 26 patients were interviewed, and 40 hours of observations conducted of the service in operation, were analysed thematically.

Results The new service implementation required co-ordinated changes in practice across multiple staff teams. Patients also needed to make changes to how they accessed the service. Multiple small 'pilots' of process changes were necessary to find workable options. This responsive model created challenges for delivering comprehensive training/communication in advance to all staff. However, staff worked together to adjust and improve the new service, and morale was buoyed through observing positive impacts on patient care. Patients valued faster results and avoiding unnecessary treatment. They were willing to drop off samples and return for a follow-up appointment the same/next day, enabling infection-specific treatment in accordance with test results thus improving antimicrobial stewardship.

Conclusions Implementation of service changes to improve access and delivery of care in the context of stretched resources can pose challenges for staff at all levels. Early evaluation of pilots of process change, provide opportunities for prompt feedback enabling adjustment, is valued. Visibility to staff of positive impacts on patient care is important in maintaining morale. The service was acceptable to patients.

\section{P041 BARRIERS AND FACILITATORS TO ANTIRETROVIRAL THERAPY INITIATION AND ADHERENCE IN INDONESIA: HEALTH CARE PROVIDER'S PERSPECTIVES}

B Hutahaean*, S Stutterheim, K Jonas. Faculty of Psychology and Neuroscience Maastricht University, Maastricht, The Netherlands

\subsection{6/sextrans-2021-sti.187}

Background Indonesia has the fourth-largest number of new HIV diagnoses per year worldwide, is the only country in the Asia-Pacific region where HIV prevalence is increasing, and the WHO aims of $90-90-90$ has not yet been reached. It is therefore important to investigate barriers and facilitators to antiretroviral (ARV) therapy initiation and adherence. This study set out to delineate these barriers and facilitators from the perspective of health care providers.

Methods Between March and May 2020, 20 semi-structured interviews were conducted with health care providers in Indonesia. Thematic analyses were subsequently conducted to ascertain categories of barriers and facilitators to initiation and adherence.

Results Main facilitators to ARV initiation and adherence were social support; good client-provider communication; less bureaucracy or easy access to ARV; and sufficient HIV and ARV knowledge among people with HIV. Additionally, the use of euphemistic terminology for ARV was a facilitator for adherence, but not for initiation; whereas having sufficient self-care motivation, a desire to live or having health goals, and HIV status acceptance were facilitators to initiation but not adherence.

Barriers to initiation and adherence included stigma; complicated bureaucracy; insufficient health care facilities, health care coverage or ARV supply; and distance to clinics. Side effects and experiencing regimens as tedious were additional barriers to adherence; and being in denial, being asymptomatic, fatalism, and the influence of anti-ARV social media were additional barriers to initiation.

Conclusion Barriers and facilitators to initiation and adherence occur on various socio-ecological levels and should therefore be targeted on structural, interpersonal, and individual levels. Health care providers can play a key role in promoting facilitators and reducing barriers, but must be supported by national and organizational level efforts that increase access to HIV clinics and health care coverage, and decrease bureaucracy and community-level initiatives that correct myths and misinformation.

\section{P042 PREVALENCE AND ASSOCIATED FACTORS OF BEING DIAGNOSED WITH SYPHILIS AMONGST MSM ATTENDING AS SEXUAL CONTACTS OF SYPHILIS}

${ }^{1,2} \mathrm{D}$ Richardson*, ${ }^{1} \mathrm{~A}$ Pickering, ${ }^{1} \mathrm{~K}$ Nichols, ${ }^{1} \mathrm{Z}$ Buss, ${ }^{1} \mathrm{D}$ Trotman, ${ }^{1} \mathrm{C}$ Fitzpatrick, ${ }^{1} \mathrm{D}$ Williams. ${ }^{1}$ Brighton and Sussex University Hospitals NHS Trust, Brighton, UK; ${ }^{2}$ Brighton and Sussex Medical School, Brighton, UK

\subsection{6/sextrans-2021-sti.188}

Partner notification strategies have increased the number of MSM attending sexually transmitted infection (STI) clinics as sexual contacts of syphilis. Current guidelines suggest testing and consideration of presumptive antimicrobial treatment. Syphilis treatment with benzathine penicillin; requires clinic resources, is painful and is associated with complications: it is important we consider strategies to rationalise presumptive antimicrobial use in MSM and promote antimicrobial stewardship.

We aimed to determine if there are any factors associated with having syphilis among MSM attending as sexual contacts of syphilis. We examined the clinical records of MSM attending as sexual contacts of syphilis from January through December 2019.

Of the 6613 MSM who attended for STI testing, 142/6613 $(2.1 \%)$ presented as sexual contacts of syphilis. The median 
age was 40 years (IQR = 31-51), 43/142 (30\%) were HIV positive, $38 / 142(27 \%)$ had been diagnosed and treated for syphilis in the past and 11/142 (8\%) presented with symptoms (possible lesions of primary or secondary syphilis). Thirteen $(9 \%, 95 \% \mathrm{CI}=4.4-13.9)$ tested positive for syphilis on the day of presentation and all were treated presumptively. MSM who were symptomatic (genital ulcer or body rash), HIV sero-positive or had a history of previous syphilis were significantly more likely to test positive for syphilis $(\mathrm{OR}=51.88$, 95\%CI3.01-893.14, $\mathrm{p}=0.007)$.

Factors associated with acquiring syphilis amongst MSM presenting as sexual contacts of syphilis were; being HIV seropositive, having a previous history of syphilis, or presenting with symptoms (possible lesions of primary or secondary syphilis).

\section{P044 TOWARDS THE SPREAD OF THE NEW L2-L2B/D-DA HYBRID VARIANT IN MEN WHO HAVE SEX WITH MEN IN FRANCE?}

${ }^{1} \mathrm{~A}$ Touati, ${ }^{1,2} \mathrm{M}$ Carrer, ${ }^{1,2} \mathrm{C}$ Bébéar, ${ }^{1,2} \mathrm{~B}$ de Barbeyrac, ${ }^{1,2} \mathrm{O}$ Peuchant*. ${ }^{1}$ Bordeaux University Hospital, Department of Bacteriology, National Reference Centre for bacterial Sexually Transmitted Infections, Bordeaux, France; '2Univ. Bordeaux, USC EA 3671, Mycoplasmal and chlamydial humans infections, Bordeaux, France

\subsection{6/sextrans-2021-sti.189}

Background Recent European studies reported lymphogranuloma venereum (LGV) cases caused by a recombinant Chlamydia trachomatis strain presenting L2-L2b/D-Da hybrid ompA sequence, which mostly affected high-risk group of men who have sex with men (MSM). Given the potential risk of dissemination of this hybrid variant, it seemed important to take stock of the situation in France.

Methods All LGV-positive anorectal specimens collected in the French National Reference Centre for bacterial Sexually Transmitted Infections (STIs) in 2018 from HIV-positive MSM with anorectal symptoms were selected. All specimens were analysed by sequencing of the ompA gene. The obtained sequences were compared by alignment with currently available chlamydial L genovars-ompA gene sequences.

Results A total of 184 LGV-positive anorectal specimens matched the selection criteria. The mean age of patients was 40.7 years [23-68]. The ompA gene was successfully sequenced for 146/184 specimens. Most specimens had ompA sequences identical to that of C. trachomatis L2 (41.7\%, 61/ $146)$ and L2b strains $(36.3 \%, 53 / 146)$. We also identified the co-circulation of several genovariants: six (4.1\%) L2-L2b/D-Da hybrid variants, two (1.3\%) L2 variants (one specimen with the A997G susbtitution and one with the G868A substitution) and $27(18.4 \%)$ L2b ompA variants (L2bv1 n=12, L2bv2 $n=3$, L2bv3 $n=1$, L2bv6 $n=5$, L2bv8 $n=1$, new L2b variant C340G $n=1$ ). We also found one specimen with L1 ompA genotype.

Conclusion The circulation of the recently described L2-L2b/ D-Da hybrid variant is sparse (4.1\%) in France whereas it represents $16.9 \%$ of LGV cases in Italy and $12.5 \%-16.5 \%$ of LGV cases in Portugal. Patient's clinical characteristics did not differ with regard to the different LGV variants. Further investigations are needed to better understand the transmission dynamics of this hybrid variant.

\section{P045 ASSESSMENT OF NEISSERIA GONORRHEA PREVALENCE AND RESISTANCE IN THE EASTERN MEDITERRANEAN REGION}

I Maatouk*. Nottingham Trent University

10.1136/sextrans-2021-sti.190

Background Neisseria gonorrhea (NG) has been identified as a high-priority public health concern because of a widespread antimicrobial resistance. The objective is to discuss the findings and implications of this emergence in the Eastern Mediterranean Region (EMR).

Methods A review of paper, reports and guidelines from the region about NG was conducted.

Results In 2015, the estimated prevalence of NG in the EMR was $0.7 \%$ for women and $0.6 \%$ for men compared to global estimations of $0.9 \%$ and $0.7 \%$ respectively. Prevalence in 2016 was higher by $0.2 \%$ for both men and women. Concerning antimicrobial resistance, regional countries from the EMR reporting data on gonococcal isolates with resistance to azithromycin, ciprofloxacin and extended-spectrum cephalosporins (ESCs) were 2 in 2009; 0 in 2010 and only 1 from 2011 to 2014 . The reported data show full susceptibility to ESCs and azithromycin and $>90 \%$ resistance to ciprofloxacin. Another report mentioned 1 reporting country in 2015 and 2016 respectively with resistances of $>90 \%$ for ciprofloxacin. Cefixime, Ceftriaxone and azithromycin resistance testing were negative. Empirical articles are very scarce. In Lebanon $(n=53)$, resistance prevalence was $38.3 \%$ to ciprofloxacin and $40.4 \%$ to azithromycin. Two papers from Morocco $(\mathrm{n}=72$ and $\mathrm{n}=135)$ reported ciprofloxacin resistance in $86.8 \%$ and $77.9 \%$ respectively in 2013 and 2018 without ceftriaxone resistance detected. A case report from Egypt was resistant to ceftriaxone and ciprofloxacin but not to azithromycin.

Discussion It is consistently clear that the EMR has the lowest number of reporting countries compared with all other regions. A call for exploration of NG epidemiology and resistance is recommended through a regional action plan to control and minimize the impact of this public health issue. The promising diagnostic technologies in NG would be beneficial for both the general and the key populations in the absence of financial support to cover current tests.

\section{P047 SEXUAL HEALTH AMONG HIV-NEGATIVE GAY AND BISEXUAL MEN IN LEBANON: A COMPARISON BETWEEN NATIVE AND IMMIGRANT/REFUGEE COMMUNITIES}

I Maatouk*, M Assi, R Jaspal. Nottingham Trent University

10.1136/sextrans-2021-sti.191

Background Unlike native gay and bisexual male (GBM), immigrants/refugees GBM have an added vulnerability to poor sexual health due to their stigmatized sexual minority status. The objective of this cross-sectional study was to compare sexual health variables in a sample of native-born and immigrants/refugees GBM in Lebanon. This study also aimed to identify the correlates of sexually transmitted infections (STIs) knowledge. 